\title{
Efficient harmony search optimization for preventive- maintenance-planning for nuclear power systems
}

\author{
Abdelkader Rami, Habib Hamdaoui, Houari Sayah, and Abdelkader Zeblah* \\ University of Djillali Liabes, Faculty of Technology, Sidi Bel Abbes, Algeria
}

Received 27 November 2012 / Accepted 23 October 2013 / Published online 26 February 2014

\begin{abstract}
This paper combines the universal generating function UGF with harmony search (HSO) meta-heuristic optimization method to solve a preventive maintenance (PM) problem for series-parallel system. In this work, we consider the situation where system and its components have several ranges of performance levels. Such systems are called multi-state systems (MSS). To enhance system availability or (reliability), possible schedule preventive maintenance actions are performed to equipments and affect strongly the effective age. The MSS measure is related to the ability of the system to satisfy the demand. The objective is to develop an algorithm to generate an optimal sequence of maintenance actions providing system working with the desired level of availability or (reliability) during its lifetime with minimal maintenance cost rate. To evaluate the MSS system availability, a fast method based on UGF is suggested. The harmony search approach can be applied as an optimization technique and adapted to this PM optimization problem.
\end{abstract}

Key words: Universal generating function harmony search, Optimization, Preventive maintenance.

\section{Nomenclature}

$C_{\mathrm{Mj}} \quad$ Minimal repair cost

$A_{\text {iv }} \quad$ Availability of $j$ th MSS devices

$h_{\mathrm{j}} \quad$ Hazard function

$Q_{\mathrm{j}} \quad$ Probability of failure of $j$ th devices

$\Xi_{\mathrm{iv}} \quad$ Performance of $j$ th devices of version $v$

$W \quad$ Demand levels

$\mathfrak{I} \quad$ Distributive operator

$\delta \quad$ Operator for parallel devices

\section{Abbreviations \\ HSO Harmony search optimization \\ PM Preventive maintenance \\ MSS Multi states system \\ UMGF Universal moment generating function}

\section{Introduction}

A necessary precondition for high production is availability of the technical equipment. In addition, reliability engineers have to build a reliable and efficient production system. The system reliability affects essentially the reliability of its equip-

*e-mail: zeblaha@yahoo.fr ments. This characteristic is a function of equipment age on system's operation life. In this work, we consider series-parallel systems. To keep the desired levels of availability, strongly performs a preventive maintenance actions to components are best than breakdown maintenance. This suggestion is supported by a number of case studies demonstrating the benefits of PM in [1]. In this case, the task is to specify how PM activity should be scheduled. One of the commonly used PM policies is called periodic PM, which specifies that systems are maintained at integer multiple of some fixed period. Another PM is called sequential PM, in which the system is maintained at a sequence of interval that have unequal lengths. The first kind of PM is more convenient to schedule. Contrary the second is more realistic when the system require more frequent maintenance at it age. A common assumption used in both these PM is that minimal repair is conducted on system if it fails between successive PM activities. In other words, minimal repairs do not change the hazard function or the effective age of the system.

Traditionally PM models assume that the system after PM is either as good as new state in this case is called perfect PM or simply replacement, as bad as old state the same as minimal repair, where he only restores the function of the system, this concept is well understood in the literature [2]. The more realistic assumption is that the system after PM not return at zero age and remains between as good as new and as bed as old. This kind of PM is called imperfect PM. The case when 
equipment fails (damage), a corrective maintenance (CM) is performed which returns equipment to operating condition, in fact specially, the task of preventive maintenance actions served to adjust the virtual age of equipment. Our particular interest is under investigation to present an harmony search algorithm which determines the optimal intervals of PM actions to minimize maintenance-cost rate or maximize mission reliability.

\subsection{Summary of previous work}

Years ago, much work was reported on policy optimization of preliminary planned PM actions with minimal repair as in $[3,4]$. Most of these researches are based on two popular approaches to determine the optimal intervals for a PM sequence. The first is reliability-based method and the second is optimization method.

In the first one the PM is performed whenever the system availability or the hazard function of the system reaches a predetermined level and the optimal PM intervals will be selected. The second is finding the optimal intervals as a decision variable in the optimization problem. [5] presents an algorithm to determine the optimal intervals based on the reliability-based method and in there models the effective age reduction and hazard function are combined. [6] presents a genetic algorithm which determine a minimal cost plan of the selecting PM actions which provides the required levels of power system reliability. A list of possible PM actions available for each MSS, are used. Each PM action is associated with cost and reduction age coefficient of its implementation.

\subsection{Approach and outlines}

The proposed approach is based on the optimization method using harmony search algorithm, which determines the intervals sequence of PM actions to minimize the maintenance-cost subject to availability or (reliability) constraints. The goal of the proposed approach is to know when, where, to which component and what kind of available PM actions among the set of available PM actions should be implemented. To evaluate the reliability and the effect of PM actions of seriesparallel MSS, UGF method is applied. It's proved to be effective at solving problem of MSS redundancy and maintenance in [7-9].

The rest of this paper is outlined as follows. We start in Section 2 with the general description of the preventive maintenance model. Next, we describe the optimization problem formulation in Section 3. A description of availability estimation based on UGF method is presented in Section 4. In Section 5, we present the harmony search algorithm. Conclusion is drawn in Section 6.

\section{Preventive maintenance}

It has been shown that the incorporation of the preventive maintenance has a benefit and success. Also it was observed that the impact of the decrease of component failure rate and improvement of component reliability is vital to maintain efficiency of production. The major subject of maintenance is

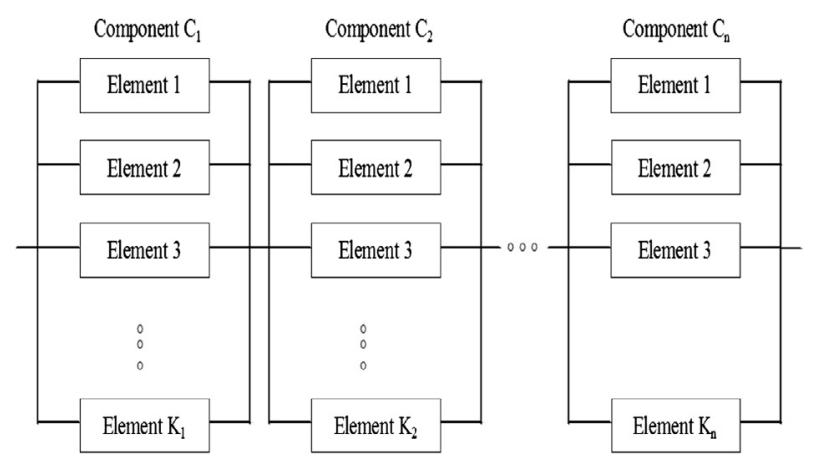

Figure 1. Series-parallel power system.

focused on the planning maintenance service of the power system. Such as cleaning, adjustment and inspection performed on operation's lifetime are classed as a preventive maintenance policy. However, all actions of PM not capable to reduce age component to zero age is imperfect. There are two main alternatives for modeling an imperfect PM activity. The first one assumes that PM is equivalent to minimal repair with probability $p$ and $1-p$ is the equivalent to replacement in [10]. The second model where the imperfect PM directly analyzes how the hazard function or the effective age change after PM as in [5]. The proposed model is based on reduction age concept. Let consider the series-parallel MSS system shown in Figure 1.

If the component $j$ undergoes on PM actions calendar at chronological times as follows:

$$
t_{j 1}, \ldots, t_{j n}
$$

Based on the second model description, the effective age after $i$-th PM actions may be written as:

$$
\begin{array}{r}
\tau_{j}(t)=\tau_{j}^{+}(t)+\left(t-t_{j i}\right) \text { for } t_{j i}<t<t_{j i+1},(1 \leq i \leq n), \\
\quad \text { and } \tau_{j}^{+}\left(t_{j i}\right)=\varepsilon_{i} \tau_{j}\left(t_{j i}\right)=\varepsilon_{i}\left(\tau_{j}^{+}\left(t_{j i-1}\right)+\left(t_{j i}-t_{j i-1}\right)\right),
\end{array}
$$

where $\tau_{j}^{+}\left(t_{j i}\right)$ is the age of component immediately after the $i$ th $\mathrm{PM}$ action which ranges in the interval $[0,1]$. By definition, we assume that $\tau_{j}(0)=0, t_{j 0}=0$ and $\varepsilon_{i}$ is the age reduction coefficient. Two limits for PM actions is, where $\varepsilon_{i}=1$ and $\varepsilon_{i}=0$. In the first case the component at least be restored to "as bed as old" state which assumes that PM does not affect the effective age. In the second case the model reduce the component age to "as good as new", which means that the component age reaches zero age (replacement). In fact, all PM actions which improve the component age are imperfect. As it be mentioned and demonstrated in [5], the hazard function of component $j$, as function of its actual age, can be calculated as:

$$
h_{j}^{*}=h_{j}\left(\tau_{j}(t)\right)+h_{j 0},
$$

where $h_{j}(t)$ is the hazard function is defined when equipment does not undergo PM actions and $h_{j 0}$ correspond to the initial age of equipment. The reliability of the equipment $j$ in the interval between PM actions $i$ and $i+1$ can be written as:

$$
\begin{aligned}
r_{j}(t) & =\exp \left(-\int_{\tau_{j}^{+}\left(t t_{j i}\right)}^{\tau_{j}(t)} h_{j}^{*}(x) \mathrm{dx}\right) \\
& =\exp \left(H_{j}\left(\tau_{j}^{+}\left(t_{j i}\right)\right)-H_{j}\left(\tau_{j}(t)\right)\right) .
\end{aligned}
$$


$H_{j}(\tau)$ represents the accumulative hazard function. Clearly if $t=t_{j i}$ in equation (4) the reliability reaches the maximum and is equal to 1 .

The Minimal repairs are performed if MSS equipment fails between PM actions, and there cost expected in interval $[0, t]$ can be given as:

$$
C_{M j}=c_{j} \int_{0}^{t} h_{j}(x) \mathrm{d} x
$$

Possible equipment $j$, undergoes PM actions at each chronological time $t_{j 1}, \ldots, t_{j n_{j}}$, in this case, the total minimal repair cost is the sum of all cost can be written as :

$$
\begin{gathered}
C_{M j}=c_{j} \sum_{i=0}^{n_{j}} \int_{\tau_{j}^{+}\left(t_{j i}\right)}^{\tau_{j}\left(t_{j i+1}\right)} h_{j}(x) \mathrm{d} x= \\
c_{j} \sum_{i=0}^{n_{j}}\left(H\left(\tau_{j}\left(t_{j i+1}\right)\right)-H_{j}\left(\tau_{j}^{+}\left(t_{j i}\right)\right)\right),
\end{gathered}
$$

where $t_{j 0}=0$ and $t_{j n_{j}+1}=T$ where $T$ represents the lifetime.

\section{Optimization problem}

Let consider a power system organized with components connected in series arrangement. Each component contains different component put in parallel. Components are characterized by their nominal performance rate $\Xi j$, hazard function $h j(t)$ and associated minimal repair cost $C j$. The system is composed of a number of failure prone components, such that the failure of some components leads only to a degradation of the system performance. This system is considered to have a range of performance levels from perfect working to complete failure. In fact, the system failure can lead to decreased capability to accomplish a given task, but not to complete failure. An important MSS measure is related to the ability of the system to satisfy a given demand.

When applied to electric power systems, reliability is considered as a measure of the ability of the system to meet the load demand $(W)$, i.e., to provide an adequate supply of electrical energy $(\Xi)$. This definition of the reliability index is widely used for power systems: see e.g., [11, 12, 13, 14, 15]. The Loss of Load Probability index (LOLP) is usually used to estimate the reliability index [16]. This index is the overall probability that the load demand will not be met. Thus, we can write $R=\operatorname{Probab}(\Xi \mathrm{MSS} \geq W)$ or $R=1-$ LOLP with LOLP $=$ $\operatorname{Probab}(\Xi M S S<W)$. This reliability index depends on consumer demand $W$.

For reparable MSS, a multi-state steady-state instantaneous availability $\mathrm{A}$ is used as $\operatorname{Probab}(\Xi M S S \geq W)$. While the multi-state instantaneous availability is formulated by equation (7):

$$
A_{\mathrm{MSS}}\{t, W\}=\sum_{\Sigma_{j} \geq D} P_{j}(t),
$$

where $\Xi$ MSS $(t)$ is the output performance of MSS at time $t$. To keep system reliability at desired level, preventive and curative maintenance can be realized on each MSS. PM actions modify components reliability and $\mathrm{CM}$ actions does not affect it. The effectiveness of each PM actions is defined by the age reduction coefficient $\varepsilon$ ranging from 0 to 1 . As in [6], the structure of the system as defined by an available list of possible PM actions ( $v$ ) for a given MSS. In this list each PM actions $(v)$ is associated with the cost of its implementation $C_{p}(v)$, and $M(v)$ is the number of equipment affected corresponding to their age reduction $\varepsilon(v)$. Commonly the system lifetime $T$ is divided into $y$ unequal lengths, and each interval have duration $\theta_{y} 1 \leq y \leq Y$, at each end of this latter an PM action is performed. This action will be performed if the MSS reliability $R(t, w)$ becomes lower than the desirable level $R_{0}$.

Let us remark that the increase in the number of intervals increases solution precision. On the other hand, the number of intervals can be limited for technical reasons.

All the PM actions performed to maintain the MSS reliability are arranged and presented by a vector $V$ as they appear on the PM list. Each time the PM is necessary to improve the system reliability; the performed following action is defined by the next number from this vector. When the scheduled PM action $v_{i}$ was insufficient to improve reliability, automatically the $v_{i+1}$ action should be performed at the same time and so on.

For a given vector $V$, the total number $n_{j}$ and chronological times of PM action in equation (1) are determined for each component $j 1 \leq j \leq J$. For all scheduled PM actions $v_{i} \in V$. The total cost of PM actions can be expressed as:

$$
C_{p}(V)=\sum_{i=1}^{N} C_{p}\left(v_{i}\right)
$$

and the cost of minimal repair can be calculated as:

$$
C_{M}(V)=\sum_{j=1}^{J} c_{j} \sum_{i=0}^{n_{j}}\left(H\left(\tau_{j}\left(t_{j i+1}\right)\right)-H\left(\tau_{j}^{+}\left(t_{j i}\right)\right)\right) .
$$

The optimization problem can be formulated as follows: find the optimal sequence of the PM actions chosen from the list of available actions which minimizes the total maintenance cost while providing the desired MSS availability. That is,

Minimize:

$$
f(V) \rightarrow C=C_{p}(V)+C_{M}(V) .
$$

Subject to:

$$
A \theta(V, D, t) \geq R_{0} .
$$

To solve this combinatorial optimization problem, it is important to have an effective and fast procedure to evaluate the availability index. Thus, a method is developed in the following section to estimate the system availability.

\section{Reliability estimation based on Ushakov's method}

The last few years have seen the appearance of a number of works presenting various methods of quantitative estimation of systems consisting of devices that have a range of working levels in $[17,18]$. Usually one considers reducible systems. In general forms the series connection, the level of working is 
determined by the worst state observed for any one of the devices, while for parallel connection is determined by the best state. However, such the approach is not applicable for the majority of real systems.

In this paper the procedure used is based on the universal $z$-transform, which is a modern mathematical technique introduced in [19]. This method, convenient for numerical implementation, is proved to be very effective for high dimension combinatorial problems. In the literature, the universal $z$-transform is also called UMGF or simply $u$-transform. The UMGF extends the widely known ordinary moment generating function [11]. The UMGF of a discrete random variable $\Xi$ is defined as a polynomial:

$$
u(z)=\sum_{j=1}^{J} P_{j} z^{\Xi_{j}} .
$$

The probabilistic characteristics of the random variable $\Xi$ can be found using the function $u(z)$. In particular, if the discrete random variable $\Xi$ is the MSS stationary output performance, the availability $\mathrm{A}$ is given by the probability $\operatorname{Proba}(\Xi \geq W)$ which can be defined as follows:

$$
\operatorname{Proba}(\Xi \geq W)=\Phi\left(u(z) z^{-W}\right),
$$

where $\Phi$ is a distributive operator defined by expressions (14) and (15):

$$
\begin{gathered}
\Phi\left(P z^{\sigma-W}\right)=\left\{\begin{array}{l}
P, \text { if } \sigma \geq W \\
0, \text { if } \sigma<W
\end{array}\right. \\
\Phi\left(\sum_{j=1}^{J} P_{j} z^{\Xi_{j}-W}\right)=\sum_{j=1}^{J} \Phi\left(P_{j} z^{\Xi_{j}-W}\right) .
\end{gathered}
$$

It can be easily shown that equations (14)-(15) meet condition $\operatorname{Proba}(\Xi \geq W)=\sum_{\Xi_{i} \geq W} P_{j}$. By using the operator $\Phi$, the coefficients of polynomial $u(z)$ are summed for every term with $\Xi \mathrm{j} \geq W$, and the probability that $\Xi$ is not less than some arbitrary value $W$ is systematically obtained.

Consider single devices with total failures and each device $i$ has nominal performance $\Xi_{i}$ and reliability $\mathrm{A}_{i}$. The UMGF of such an device has only two terms can be defined as:

$$
u_{i}(z)=\left(1-A_{i}\right) z^{0}+A_{i} z^{\Xi_{i}}=\left(1-A_{i}\right)+A_{i} z^{\Xi_{i}} .
$$

To evaluate the MSS availability of a series-parallel system, two basic composition operators are introduced. These operators determine the polynomial $u(z)$ for a group of devices.

Parallel devices: let consider a system device $m$ containing $\mathrm{Jm}$ devices connected in parallel. The total performance of the parallel system is the sum of performances of all its devices. In power systems, the term capacity is usually used to indicate the quantitative performance measure of an device in [20]. Examples: generating capacity for a generator, carrying capacity for an electric transmission line, etc. Therefore, the total performance of the parallel unit is the sum of capacity (performances) in [19]. The $u$-function of MSS device $m$ containing Jm parallel devices can be calculated by using the $\mathfrak{I}$ operator:

$u_{p}(z)=\mathfrak{I} \quad{ }_{n}\left(u_{1}(z), \quad u_{2}(z), \ldots, \quad u_{n}(z)\right), \quad$ where $\mathfrak{I}\left(\Xi_{1}\right.$, $\left.\Xi_{2}, \ldots, \Xi_{n}\right)=\sum \Xi_{i}$.

Therefore för a pair of devices connected in parallel:

$$
\begin{aligned}
\mathfrak{J}\left(u_{1}(z), u_{2}(z)\right. & =\mathfrak{J}\left(\sum_{i=1}^{n} P_{i} z^{a_{i}}, \sum_{j=1}^{m} Q_{j} z^{b_{j}}\right) \\
& =\sum_{i=1}^{n} \sum_{j=1}^{m} P_{i} Q_{j} z^{a_{i}+b_{j}} .
\end{aligned}
$$

The parameters $a_{i}$ and $b_{j}$ are physically interpreted as the performances of the two devices. $n$ and $m$ are numbers of possible performance levels for these devices. $P_{i}$ and $Q_{j}$ are steadystate probabilities of possible performance levels for devices. One can see that the $\mathfrak{I}$ operator is simply a product of the individual $u$-functions. Thus, the device UMGF is: $u_{p}(z)=\prod_{j=1}^{J_{m}} u_{j}(z)$. Given the individual $\mathrm{JMGF}_{m}$ of devices

defined in equation (11), we have: $u_{p}(z)=\prod_{j=1}^{J_{m}}\left(1-A_{j}+A_{j} z^{\Xi_{i}}\right)$.
Series devices: when the devices are connected in series, the device with the least performance becomes the bottleneck of the system. This device therefore defines the total system productivity. To calculate the $u$-function for system containing $n$ devices connected in series, the operator $\delta$ should be used: $u_{s}(z)=\delta\left(u_{1}(z), \quad u_{2}(z), \ldots, u_{m}(z)\right)$, where $\delta\left(\Xi_{1}, \Xi_{2}, \ldots\right.$, $\left.\Xi_{m}\right)=\min \left\{\Xi_{1}, \Xi_{2}, \ldots, \Xi_{m}\right\}$ so that

$$
\begin{aligned}
\delta\left(u_{1}(z), u_{2}(z)\right) & =\delta\left(\sum_{i=1}^{n} P_{i} z^{a_{i}}, \sum_{j=1}^{m} Q_{j} z^{b_{j}}\right) \\
& =\sum_{i=1}^{n} \sum_{j=1}^{m} P_{i} Q_{j} z^{\min \left\{a_{i}, b_{j}\right\}} .
\end{aligned}
$$

Applying composition operators $\mathfrak{I}$ and $\delta$ consecutively, one can obtain the UMGF of the entire series-parallel system. To do this we must first determine the individual UMGF of each device.

Devices with total failures: let consider the usual case where only total failures are considered and each subsystem of type $i$ and version $v i$ has nominal performance $\Xi_{i v}$ and availability $A_{i v}$. In this case, we have: $\operatorname{Proba}\left(\Xi=\Xi_{i v}\right)=A_{i v}$ and $\operatorname{Proba}(\Xi=W)=1-A_{i v}$ The UMGF of such an device has only two terms can be defined as in equation (11) by $u_{i}^{*}(z)=\left(1-A_{i v}\right) z^{0}+A_{i v} z^{\Xi_{i v}}=1-A_{i v}+A_{i v} z^{\Xi_{i v}}$. Using the $\mathfrak{I}$ operator, we can obtain the UMGF of the $i$-th system device containing $k_{i}$ parallel devices $u_{i}(z)=\left(u_{i}^{*}(z)\right)^{k_{i}}=\left(A_{i v} z^{\Xi_{i v}}+\right.$ $\left.\left(1-A_{i v}\right)\right)^{k_{i}}$.

The UMGF of the entire system containing $n$ devices connected in series is:

$$
u_{s}(z)=\delta\left(\begin{array}{c}
\left(A_{1 v} z^{\Xi_{1 v}}+\left(1-A_{1 v}\right)\right)^{k_{1}}, \\
\left(A_{2 v} z^{\Xi_{2 v}}+\left(1-A_{2 v}\right)\right)^{k_{2}}, \ldots, \\
\left(A_{n v} z^{\Xi_{n v}}+\left(1-A_{n v}\right)\right)^{k_{n}}
\end{array}\right) .
$$




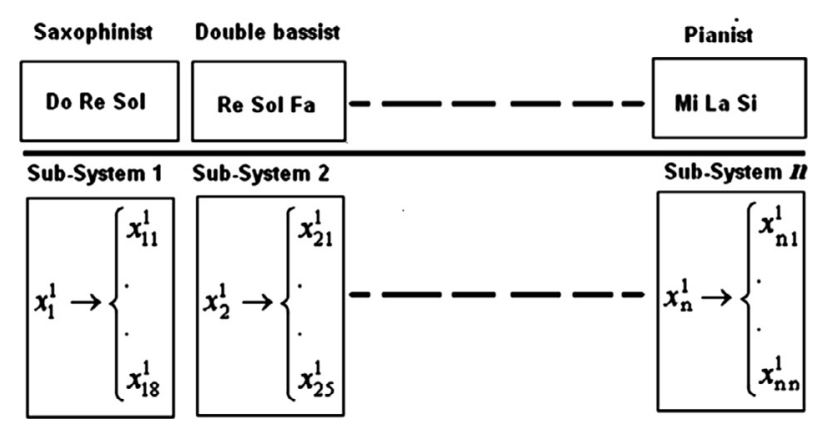

Figure 2. Synoptic modeling HS for optimization.

To evaluate the probability $\operatorname{Proba}(\Xi \geq W)$ for the entire system, the operator $\Phi$ is applied to equation (18):

$$
\operatorname{Proba}(\Xi \geq W)=\Phi\left(u_{s}(z) z^{-W}\right) .
$$

\section{The harmony search approach}

The problem formulated is a complicated NP-hard complex problem. The total number of different solutions to be examined is very large. An exhaustive examination of the enormous number of possible solutions is not feasible given reasonable time limitations. Thus, because of the search- space size of the problem. Adopting the idea that existing evolutionary or meta-heuristic algorithms are found in the paradigm of natural processes, a new algorithm can be conceptualized from a musical performance process (say, a jazz trio) in $[21,22]$ involving searching for a better harmony. Musical performance seeks a best state (fantastic harmony) determined by aesthetic estimation, as the optimization process seeks a best state (global optimum: minimum cost; minimum error; maximum benefit; or maximum efficiency) determined by objective function evaluation. Aesthetic estimation is done by the set of the pitches sounded by joined instruments, as objective function evaluation is done by the set of the values produced by composed variables; the aesthetic sounds can be improved practice after practice, as the objective function values can be improved iteration by iteration in [23].

Figure 2 shows the structure of the Harmony Memory (HM) that is the core part of the HS algorithm. Consider a jazz trio composed of saxophone, double bass, and guitar. There exist certain amount of preferable pitches in each musician's memory: saxophonist, \{Do, Fa, Mi, Sol, Re $\}$; double bassist, $\{\mathrm{Si}, \mathrm{Do}, \mathrm{Si}, \mathrm{Re}, \mathrm{Sol}\}$; and guitarist, $\{\mathrm{La}, \mathrm{Sol}, \mathrm{Fa}, \mathrm{Mi}, \mathrm{Do}\}$. If saxophonist randomly plays $\{\mathrm{Sol}\}$ out of its memory $\{\mathrm{Do}$, $\mathrm{Fa}, \mathrm{Mi}, \mathrm{Sol}, \mathrm{Re}\}$, double bassist $\{\mathrm{Si}\}$ out of $\{\mathrm{Si}, \mathrm{Do}, \mathrm{Si}, \mathrm{Re}$, Sol $\}$, and guitarist $\{\mathrm{Do}\}$ out of $\{\mathrm{La}, \mathrm{Sol}, \mathrm{Fa}, \mathrm{Mi}, \mathrm{Do}\}$, the new harmony (Sol, Si, Do) becomes another harmony (musically chord). And if this new harmony is better than existing worst harmony in the HM, the new harmony is included in the HM and the worst harmony is excluded from the HM [24]. This procedure is repeated until fantastic harmony is found.

\section{Harmony search algorithm}

Step 1. Initialize:

Set $\quad \mathrm{N}$ istrument: $=\mathrm{N}$ _sybsystem $\{\mathrm{N}$ is the integer number\},

Set MHCR: $=0.7$ harmony memory considering rate $\}$,

Set PAR: $=0.35$ Pitch adjustment rate $\}$,

Set PAD \{Pitch adjustment decision\},

Set NI:=75 improvisation number $\}$,

Set $\mathrm{t}:=0\{t$ is the time counter $\}$,

Set Interval_t $:=0\{\theta$ is Interval time $\}$,

Set List PM_actions $\left[\mathrm{L}_{\mathrm{n} 1}\right]:=\quad\left[P_{n 1}^{\mathrm{v}}\right]$ \{Available PM actions\},

Set PM_Action: $=0\{m$ is the time counter $\}$,

For every Components $(\mathrm{i}, \mathrm{j})$ set an initial value $x_{i j}^{1} \rightarrow\left\{x_{11}^{1}, \ldots, x_{n 1}^{1}\right\} x_{i j}^{1}$,

Set For All components $(1 \leq j \leq J)$ :

Set Effectve_age: $=\tau \mathrm{j}$

Set $\mathrm{H}_{\mathrm{j}}\left(\tau_{\mathrm{j}}^{+}\right)=0$

For $\mathrm{i}:=1$ to $\mathrm{n}$ do

$\mathbf{M a t}_{\mathrm{k}}(\mathrm{HM}):=\mathrm{i}$ \{starting component is the first element of the Mat list of the $k$ th instrument $\}$,

The HM matrix is filled with randomly generated as the HMS.

Step 2. Improvise a New list $\mathbf{M a t}_{\mathrm{k}}$ until is full \{this step will be repeated $(n-1)$ times $\}, \quad x^{n v} \rightarrow$ $\left\{x_{11}^{n v}, x_{12}^{n v}, \ldots, x_{n k}^{n v}\right\}$

2.0. $\mathrm{y}:=$ Interval time +1

2.1. $\mathrm{T}:=$ Interval time $+\mathrm{t}$ '

2.2. Effectve_age: $=\tau \mathbf{j}+\mathrm{t}$

2.3. New PM_action: $=x^{n v} \in\left[P_{n 1}^{\mathrm{v}}\right]$

Step 3. Compute For $\mathrm{j}:=1$ To $\mathrm{J}$

$\mathrm{H}_{\mathrm{j}}\left(\tau_{\mathrm{j}}\right)$ According equation (3)

End

Compute For $\mathrm{j}:=1$ To $\mathrm{J}$

$r_{j}\left(\tau_{j}\right)$ According equation (4)

Step 4. Compute For $\mathrm{j}:=1$ To $\mathrm{n}$ do \{for every $k$ th instrument on Subsystem i\}

Choose the PM_Action with probability

$P A D$ for $x_{i j}^{n v}\left\{\begin{array}{l}\bar{Y} e s \text { If } \operatorname{ran}_{1} \leq P A R \\ \text { No If } \operatorname{ran}_{1} \succ(1-P A R)\end{array}\right.$

Then $x_{i j}^{n v} \leftarrow x_{g i}^{n v} \pm \operatorname{ran}_{i}() * b w$

With Cost j, corresponding to Equation (8) and (9)

Increment to the $k$ th instrument on the $i$ subsystems

Insert PM_Action and Cost $\mathrm{j}$ in $\mathbf{M A T}_{\mathrm{k}}$ (s).

Step 5. If $\mathrm{R}^{-}(\mathrm{t}, \Xi)<\mathrm{R}_{0}$ increment and define the new PM_Action to perform, add the new cost to total Cost. Recalculate the reliability $\mathrm{r}$

Else Goto Step 2.

If $\mathrm{R}(\mathrm{t}, \Xi)>=$ evaluate the cost of minimal repair for all components $(1 \leq j \leq J)$ : and add these costs to the total cost.

Print minimal total cost to the corresponding reliability and Stop.

\section{Illustrative example}

Let consider a series-parallel MSS (Nuclear power systems) consisting of five subsystems connected in series arrangement 


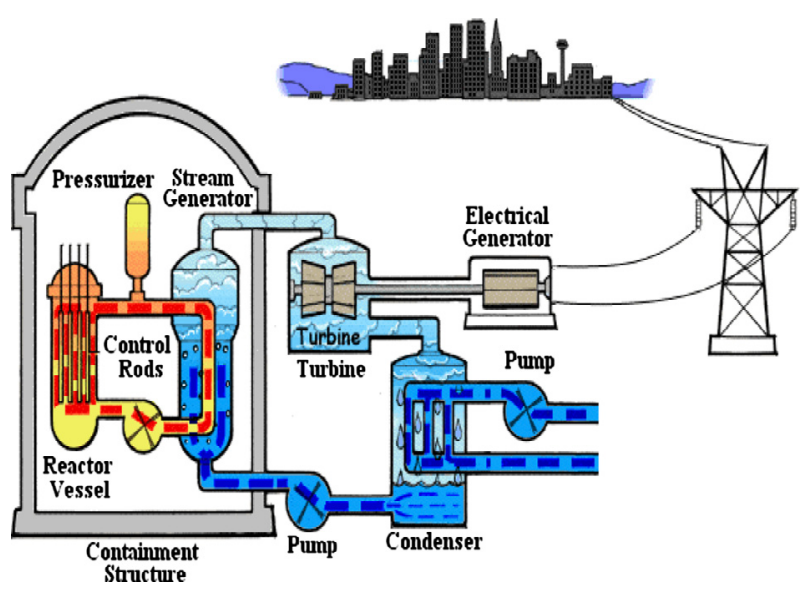

Figure 3. Detailed series-parallel nuclear power system.

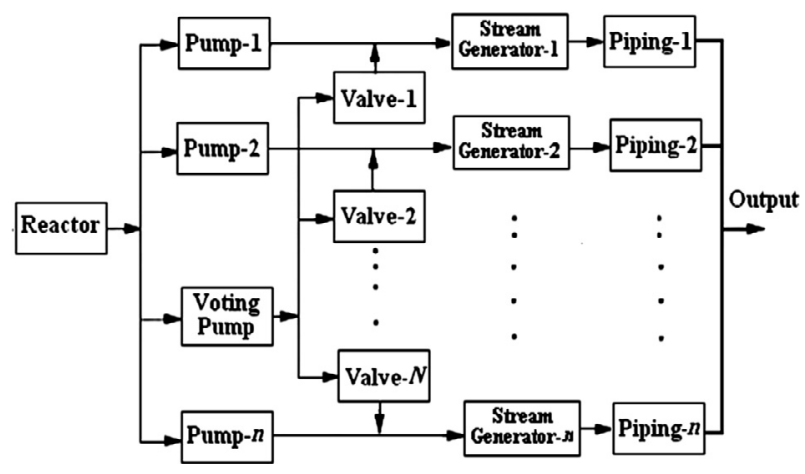

Figure 4. Synoptic of series-parallel nuclear power system.

Table 1. Parameter of components.

\begin{tabular}{lccccc}
\hline & $\lambda$ & $\delta$ & $h_{0}$ & Min_cost & $\Xi \%$ \\
\hline 1 & 0.050 & 1.8 & 0.001 & 1.02 & 97 \\
2 & 0.050 & 1.8 & 0.003 & 0.90 & 80 \\
3 & 0.050 & 1.8 & 0.003 & 0.90 & 80 \\
4 & 0.050 & 1.8 & 0.003 & 0.90 & 80 \\
5 & 0.020 & 1.3 & 0.002 & 0.70 & 70 \\
6 & 0.020 & 1.3 & 0.002 & 0.60 & 70 \\
7 & 0.020 & 1.3 & 0.002 & 0.70 & 70 \\
8 & 0.080 & 1.6 & 0.006 & 0.80 & 85 \\
9 & 0.070 & 1.8 & 0.005 & 0.60 & 89 \\
10 & 0.050 & 1.8 & 0.003 & 0.80 & 90 \\
11 & 0.080 & 1.7 & 0.002 & 0.80 & 90 \\
12 & 0.030 & 1.6 & 0.001 & 0.90 & 95 \\
13 & 0.004 & 1.5 & 0.001 & 0.80 & 87 \\
14 & 0.010 & 1.6 & 0.003 & 0.85 & 80 \\
15 & 0.200 & 1.6 & 0.005 & 0.75 & 92 \\
\hline
\end{tabular}

as depickted in Figures 3 and 4. The system contains 15 equipments with different performance and reliability (parameters) as given in Tables 1 and 2, the process is done with basic components to transmit the stream energy to the electrical generator. The reliability of each component is defined by veibull function: $h(t)=\lambda^{\delta} \delta(\tau(t))^{(\delta-1)}+h_{0}$.
Table 2. Parameter of PM_actions.

\begin{tabular}{llc}
\hline PM_actions & \multicolumn{1}{c}{ Components } & PM_cost $\$ 103$ \\
\hline 01 & Reactor & 10.2 \\
02 & Pump-1 & 02.9 \\
03 & Pump-2 & 02.8 \\
04 & Pump-3 & 02.8 \\
05 & Voting-pump & 03.6 \\
06 & Servo-valve-1 & 02.7 \\
07 & Servo-valve-2 & 02.7 \\
08 & Servo-valve-3 & 02.7 \\
09 & Stream-generator-1 & 08.1 \\
10 & Stream-generator-2 & 08.1 \\
11 & Bi-stream-generator & 07.5 \\
12 & Pipe-1-2-3-4 & 02.7 \\
\hline
\end{tabular}

Table 3. The best PM plan for $R(t, 0.82)>0.9$.

\begin{tabular}{lccc}
\hline$t$ & PM_Actions & Affected components & $R(t, 0.82)$ \\
\hline 12.250 & 05,00 & $(4)$ & 0.923 \\
16.652 & 08,00 & $(6,7,8)$ & 0.922 \\
20.000 & 09,10 & $(9,10)$ & 0.954 \\
22.110 & 04,00 & $(10 ; 11)$ & 0.911 \\
25.640 & 12,00 & $(13,14,15)$ & 0.937 \\
\hline
\end{tabular}

MSS lifetime is 10 years. The time for possible PM actions are spaced intervals of $\theta=1.5$ months as given in Table 3 . The problem is to guarantee a PM plan which provide the system work during its lifetime with a performance, reliability not less than $\Xi 0, R_{0}$ and the age reduction is the same of all components $\varepsilon=0.56$.

\section{Conclusion}

In this paper we formulated the problem of imperfect maintenance optimization for series-parallel nuclear power system structure. This work focused on selecting the optimal sequence of intervals to perform PM actions to improve the availability. The model analyzes cost and reliability, to construct a strategy to select the optimal maintenance intervals, formulating a complex problem. An exhaustive examination of all possible solution is not realistic, considering reasonable time limitations. Because of this, an efficient meta-heuristic can be applied (Harmony Search Algorithm) to solve the formulated problem. More specifically, the harmony search approach is a good solution for such a combinatorial problem.

\section{References}

1. Gude D, Schmidt KH. 1993. Preventive maintenance of advanced manufacturing systems: a laboratory experiment and its implications for human centered approach. International Journal of Human Factors in Manufacturing, 3, 335-350.

2. Brown M, Proschan F. 1983. Imperfect repair. Journal of Applied probability, 20, 851-859.

3. Zhao M. 2003. On preventive maintenance policy of critical reliability level for system subject to degradation. Reliability Engineering \& System Safety, 79(3), 301-308. 
4. Borgonovo E, Marseguerra M, Zio E. 2000. A Monte Carlo methodological approach to plant availability modeling with maintenance, aging and obsolescence. Reliability Engineering \& System Safety, 67(1), 61-73.

5. Lin D, Zuo MJ, Yam RCM. 2000. General sequence imperfect preventive maintenance models. International Journal of Reliability, Quality and Safety Engineering, 7(3), 253-266.

6. Levitin G, Lisniaski A. 2000. Optimization of imperfect preventive maintenance for multi-state systems. Reliability Engineering \& System Safety, 67, 193-203.

7. Monga A, Toogood R, Zuo MJ. 1997. Reliability-based design of systems considering preventive maintenance and minimal repair. International Journal of Reliability, Quality and Safety Engineering, 4, 55-71.

8. Levitin G, Lisniaski A. 1999. Optimal multistage modernization of power system subject to reliability and capacity requirements. Electric Power System Research, 50, 183-190.

9. Ushakov IA, Levitin G, Lisnianski A. 2002. Multi-state system reliability: from theory to practice. Proc. of 3 Int. Conf. on Mathematical Methods in Reliability, MMR 2002, Trondheim, Norway, p. 635-638.

10. Nakagawa T. 1999. Sequential imperfect preventive maintenance policies. IEEE Transactions on Reliability, 37(3), 295298.

11. Ross SM. 1993. Introduction to probability models. Academic press.

12. Murchland J. 1975. Fundamental concepts and relations for reliability analysis of multi-state systems, in Reliability and Fault Tree Analysis, Barlow R, Fussell J, Singpurwalla N, Editors. SIAM: Philadelphia.

13. Levitin G, Lisnianski A, Ben-Haim H, Elmakis D. 1998. Redundancy optimization for series-parallel multi-state systems. IEEE Transactions on Reliability, 47(2), 165-172.
14. Levitin G, Lisniaski A, Ben-haim H, Elmakis D. 1996. Power system structure optimization subject to reliability constraints. Electric Power System Research, 40, 145-152.

15. Levitin G, Lisnianski A, Ben-Haim H, Elmakis D. 1997. Structure optimization of power system with different redundant elements. Electric Power Systems Research, 43(1), 19-27.

16. Billinton R, Allan R. 1990. Reliability evaluation of power systems. Pitman. IEEE Transactions PWRS-S, 1-11.

17. Reinschke B. 1985. System of elements with many states. Radio i svyaz: Moscow.

18. El-Neweihi E, Proschan F. 1984. Degradable systems: a survey of multistates system theory. Communications in Statistics Theory and Methods, 13(4), 405-432.

19. Ushakov IA. 1986. Universal generating function. Soviet Journal of Computer and System Sciences, 24(5), 118-129.

20. Chern MS. 1992. On the computational complexity of reliability redundancy allocation in a series system. Operations Research Letters, 11, 309-315.

21. Geem ZW, Tseng C-L, Park Y. 2005. Harmony search for generalized orienteering problem: best touring in China. Book Advanced in Natural Computation, vol. 361, Springer: Berlin/ Heidelberg, p. 741-750.

22. Mahdavi M, Fesanghary M, Damangir E. 2007. An improved harmony search algorithm for solving optimization problems. Applied Mathematics and Computation, 188, 1567-1579.

23. Fesanghary M, Mahdavi M, Minary-Jolandan M, Alizadeh Y. 2008. Hybridizing harmony search algorithm with sequential quadratic programming for engineering optimization problems. Computer Methods in Applied Mechanics and Engineering, 197, 3080-3091.

24. Omran MGH, Mahdavi M. 2008. Global-best harmony search. Applied Mathematics and Computation, 198, 643-656.

Cite this article as: Rami A, Hamdaoui H, Sayah H \& Zeblah A: Efficient harmony search optimization for preventive-maintenanceplanning for nuclear power systems. Int. J. Simul. Multisci. Des. Optim., 2014, 5, A17. 